Elvira Margherita Ghirlanda

\title{
«Compagna Chiang Ch'ing, le rivoluzioni tradiscono sempre, come i figli...». Mela o Della dialettica del Canone in Dacia Maraini. ${ }^{1}$
}

\begin{abstract}
Attraverso il dramma Mela di Dacia Maraini si intende riflettere sul difficile rapporto della scrittrice col canone (in questo caso, teatrale) e sulla funzione dialettica che in termini culturali esso può assumere. La pièce ha diversi livelli di lettura: l'azione in cui le protagoniste (la nonna Mela, la figlia Rosaria e la nipote Carmen) si confrontano; l'allegorizzazione delle loro dinamiche nella biografia di un personaggio, assente sulla scena, ma costante referente - Chiang Ch'ing, moglie di Mao, ricordata da Maraini anche come attrice e drammaturga della Rivoluzione Culturale Cinese; infine la ricorrenza nel testo di una riflessione metateatrale/culturale sul rapporto col Canone, fulcro simbolico dell'intero spettacolo.
\end{abstract}

\section{Un sogno di inadeguatezza e afasia}

Ogni generazione è costretta a rielaborare una sua interpretazione e un suo originale rapporto con la memoria. Non ci sono regole, ovvero le regole ce le costruiamo volta per volta, secondo la sensibilità sociale e psicologica. Le regole sono necessarie, non se ne può fare a meno, però non possono essere trascendentali, calate dall'alto o stabilite una volta per tutte, ma devono avere in sé la possibilità di modificarsi (MARAINI 2010, 379).

All'interno del saggio Inadeguatezza - di questo «piccolo scritto autobiografico» (ibid., 377) - Dacia Maraini ci consegna l'ammissione di un disagio personale da sempre avvertito, che immediatamente confessa l'espansione in una dimensione comune: «da dove deriva il sentimento di inadeguatezza femminile?» (ivi). L'autrice trova risposta, ovviamente, non in ragioni di ordine "naturale", quanto nella reazione sommessa delle donne a dinamiche storico-sociali attinenti all'istituzione di valori e regole e, conseguentemente, al relativo spazio concesso alla libertà. Il termine "inadeguatezza", infatti, etimologicamente si racconta come negativo dell'intensivo di "equo" (rendere uguale) e contiene in sé l'assunzione di valori e paradigmi; rimanda quindi fortemente alla cognizione di

The e-journal «altrelettere» is hosted at the URL: http://www.altrelettere.uzh.ch , in accordance with the Open Access Policy of the University of Zurich. Please cite this article as follows: Elvira Margherita GHIRLANDA, "Compagna Chiang Ch’ing, le rivoluzioni tradiscono sempre, come i figli...». Mela o Della dialettica del Canone in Dacia Maraini in «altrelettere», 21.03.2018, DOI: 10.5903/al_uzh-38.

(C) This article is licensed under a Creative Commons Attribution 2.5. Switzerland (CC BY-NC-ND 2.5). Please read the license terms on the website: http://creativecommons.org/licenses/by-nc-nd/2.5/ch/deed.en 
"autorità" e al rapporto dialettico tra possibilità e impossibilità di conciliazione con essa, nonché all'archetipo dell'autore.

L'autrice tenta di risolvere e sanare tale conflitto mettendo in relazione il vincolo delle regole con la potenza della memoria e ponendo trasversalmente una riflessione retrospettiva su alcune sue esperienze biografiche (come la reclusione nel campo di concentramento giapponese) e letterarie, nello specifico narrative.

Partendo, infatti, da posizioni bergsoniane Maraini definisce la memoria come la nostra coscienza, o meglio come il rapporto col passato e la capacità di interpretare ed elaborare questo tramite la messa in relazione e l'accordo tra un tempo personale e uno collettivo. E ancora, la «memoria è [...] l'osservazione del passaggio del tempo» (ibid., 380), il rapportarsi con «il più grande mistero con cui noi ci troviamo a confrontarci» (ivi), poiché non ci si può sottrarre dal constatare che il tempo diventa trascorso per una condizione ignota, ma necessaria all'atto stesso di esserci. Precisamente da questo arcano si sviluppa per Maraini ogni sua tensione narrativa, come tentativo di risposta e nel contempo di infinita interrogazione, e soprattutto il romanzo, il genere che elabora il tempo come "liquidità". Tuttavia - per questo ricomporre i processi identificativi e autofondanti dell'io e della società con gli assunti diacronici e con il paradigma dell'autorità - tali riflessioni non possono esimersi dall'interrogare la produzione teatrale di Maraini, là proprio dove il tempo diviene atto mediante una azione che è parola.

Come la necessità narrativa scaturisce, infatti, da un disagio rispetto al modello, così quella rappresentativa risponde a una seconda espressione dell'inadeguatezza, l'afasia:

Spesso faccio questo sogno: sono sopra un palcoscenico [...] devo dire una battuta ma non mi viene, ce l'ho sulla punta della lingua ma mi sfugge. [...] Cerco freneticamente, cerco nella memoria la battuta che pure so di conoscere e che ho già ripetuto tante volte ma non riesco a trovarla. [...] Forse il teatro serve a questo, a vincere una paura profonda, ridare la parola ad un silenzio primordiale che ancora minaccia i nostri sogni infantili» (MARAINI 2000, V).

Integrata in una dimensione temporale non più unicamente liquida, ma ciclica, la memoria assurge a storia che concede l'eterno ritorno dell'uguale, ma che, 
proprio in virtù di questa facoltà, è anche in potere di negarlo. Infatti l'övo $\mu \alpha$, nel suo continuo essere reiterato, rischia per usura di piombare nel magma indistinto di una Babele originaria e poi, ancora più a fondo, nella vacuità del significato, trascinando con sé, in questa condizione di non-essere, il parlante. Da qui la ricerca dell'autrice di una parola che non sia solo «strumento di indignazione» (MARINELli-MATASSA 2008, 14), ma sia anche in grado di agire sulla scena, di esercitare la sua forza e poi consumarsi apparentemente, tramutandosi invece in memoria e, paradossalmente, in avvenire. Una ossimorica memoria profetica che sussume l'antico archetipo di una razionalità ordinatrice (voũs):

Per me il teatro rimaneva nonostante tutto il luogo in cui ci si interroga sul futuro, sullo sguardo di Dio: il Signore ci osserva o siamo noi a osservare lui e quindi a inventarlo di sana pianta? E questo sguardo, inventato o vero che sia, è uno sguardo d'amore o di odio o peggio ancora di indifferenza? E che posto ha la madre di tutte le madri in questa cosmogonia? (MARAINI 2000, VI).

La rievocazione, come sottrazione all'oblio, esercita propriamente la funzione di accesso alla dialettica tra sé e altro, tra uomo e storia, tra norma e legge, affrontando, tacitamente, il contrasto tra uomo e Cronos: circuiti che fondano le strutture intime di una poetica assetata di ideologia - ancora prima di divenire politica, femminista, sociale o militante.

\section{Mela e le declinazioni di un conflitto titanico}

A tal proposito, agli inizi degli anni '8o, si colloca emblematicamente il dramma Mela (1981), rappresentato per la prima volta il 17 gennaio $1982 .{ }^{2}$ Inaugurata da Maria Stuarda (1980), un testo che in maniera inequivocabile pone l'attenzione sulla dinamica "donne e potere", questa nuova stagione drammaturgica attinge «dalla realtà, dalla storia, dalla letteratura, animata da una sensibilità spontanea di tipo aristotelico» (CRUCiATA 2003, 36). Trascorse, ma non superate, le esperienze degli anni '60 e '70 - definite rispettivamente da Maraini «teatro povero» e «teatro di barricata» (MARAINI 2000, 701) - Mela è una commedia in due atti che affronta, in diverse declinazioni e profondità, la complessità del rapporto con il modello. 


\subsection{Il gatto Tarallino, l'aneddoto di un conflitto generazionale}

Mela, Rosaria e Carmen: tre donne di sessanta, quaranta e diciannove anni; tre donne e una famiglia, poiché Mela è madre di Rosaria che a sua volta è la madre di Carmen. Ancora tre donne e una cucina, unico ambiente in cui si sviluppa la pièce; e infine tre donne e un uomo, Costante, assente dalla scena, ma determinante nello svolgimento della vicenda.

L'azione si compie in un'epoca contemporanea, come si evince da espliciti rimandi a vicende storiche, e in una città del Sud d'Italia, basti notare i nomi dei personaggi, geograficamente connotati in maniera evidente. I tre caratteri sono centrati e delineati fin dalle prime battute. Mela è una personalità solida e definita, libera. Nel suo passato di suggeritrice teatrale ha girato l'Italia in tournée; adesso, anche da ferma, alle soglie della vecchiaia, decide di vivere la sua quotidianità in un perenne viaggio, come se fosse «alla stazione» (MARAINI 2001, 52). Pur consapevole della sua età e di un tempo che potrebbe recidersi, sceglie di non combatterlo, ma di accoglierlo godendone: «Aspetto il treno. Intanto mi guardo intorno, mi diverto, gioco, ballo come si fa alla stazione» (ivi). È una «cinica col senso dell'umorismo» (MARAINI-Murrali 2013, 61), e così si porta a ballare gli amici di Carmen, fuma erba, seduce i ragazzi della nipote, fa tardi la notte, non lascia all'esperienza nulla di inesplorato. Rosaria è un'ex sessantottina, «è una donna forte, che lavora seriamente» (ivi) come traduttrice

e mantiene tutta la famiglia. La sua debolezza sta nella cecità idealistica. Crede nell'amore eterno, crede nella rivoluzione pura, crede nell'amicizia, nella fedeltà e naturalmente casca dentro le trappole più prevedibili (ivi).

Ha un matrimonio fallito alle spalle, che però le ha lasciato una giovane figlia, e una attuale relazione con Costante, uomo con cui ha condiviso ideali ed esperienze politiche. Carmen, visibilmente irrisolta, si autodefinisce, con lucidità, «viziata, pigra, egoista, noiosa, capricciosa» (MARAINI 2001, 82); è la più giovane delle tre, figlia di Rosaria e nipote di Mela e in virtù di questa giovinezza «si sente in diritto [...] di prendere tutto e subito infischiandosene del dolore altrui» (MARAINI-MurRALI 2013, 61). Ha intrapreso infatti, già da due anni, una 
relazione nascosta con Costante, e mantiene parallelamente ulteriori legami d'amore, come con Mario, da «sei mesi» il suo «ragazzo» (MARAINI 2001, 71-72). Aspetta un figlio - sembrerebbe da poco tempo, visto che riesce a tenere inosservata in casa la sua situazione - probabilmente da Costante e ciò la mette nella condizione di doverne parlare con la madre. L'azione teatrale, tra equivoci e un drammatico cinismo, si gioca quindi intorno alla confessione di Carmen a Rosaria, in un contesto "estremo» che risulterà «in ultima analisi normale» (MARIANI 2000, 66).

Il conflitto tra le due però, come si diceva, si trasforma in un conflitto generazionale tra madre e figlia (nelle due diverse coppie) e tra nonna e nipote. Da qui l'effetto antifrastico dell'ambientazione in cucina: simbolo (per metonimia) del legame familiare in quanto luogo di condivisione non solo del rito d'aggregazione primario, ma anche di un gesto vitale quale il nutrire. La cucina di Rosaria però non nutre e non condivide, anzi, proprio in virtù di quell'originario valore culturalmente affermato, evidenzia la sua inesorabile deriva antifrastica. ${ }^{3}$

Rosaria è l'unico personaggio che prepara da mangiare in famiglia, e lo fa quindi per sé e per le altre.

Dal momento in cui un bisogno viene preso in carico dalle norme di produzione e di consumo (in poche parole, dal momento in cui passa al rango di istituzione), in esso non è più possibile dissociare la funzione dal segno della funzione reale (BARTHES 1998, 34)

Così cibo e cucina in Mela divengono segno di una lotta intestina tra le protagoniste preannunciata ed eclatante; un focus privilegiato per una prospettiva di analisi che illustri gli immaginari dei tre personaggi.

Carmen, vegetariana, si rifiuta di mangiare il cibo preparato dalla madre; Rosaria legge nella scelta della figlia solo una delle sue innumerevoli «fisime» (MARAINI 2001, 47) e ne denuncia le contraddizioni:

CARMEN: Il fatto che non usi le tue mani per uccidere non ti assolve dal delitto contro quelle povere bestie. Tu mangi la carne straziata da qualcun altro.

$[\ldots]$ 
ROSARIA: Vedi che anche tu... porti i guanti fatti con la pelle degli esseri viventi. Scarpe, borse, cinture...

CARMEN: Io non li mangio. Tu li ingurgiti, io mi limito a calzarli.

ROSARIA: Tu non hai sofferto la fame, il freddo...

CARMEN: ̀̀ un gioco vostro, che non mi riguarda.

ROSARIA: Tu vivi a carico e ti puoi permettere di fare la schizzinosa.

(ibid., 48)

I termini della dialettica tra le due sono già collocati da questa prima scena. Il cibo appare pretesto di scontro ideologico in cui collimano la società del benessere di Carmen (che si può permettere di «fare la schizzinosa») e quella bellico-rivoluzionaria di Rosaria. Singolare in merito la posizione straniante di Mela che successivamente commenterà il vegetarianismo di Carmen con il racconto - dal sapore di un aneddoto - del gatto Tarallino:

MELA: Un po' di spezzatino?

CARMEN: Non mangio cadaveri!

MELA: Te l'ho raccontato di quando ho mangiato un gatto al forno con le patatine? [...] Mica lo sapevo che era un gatto. Quel coglione di mio cognato dice: è coniglio, mangia. Io taglio, mastico. Un po' dolce questo coniglio, ma che ci hai messo, lo zucchero?... Era il '45... In giro non c'era niente da mettere in bocca... Lui scoppia a ridere e dice: hai mangiato il gatto e non te ne sei neanche accorta! Quale gatto? Dico, io. Tarallino, il tuo preferito. Gli ho tirato un coltello che l'ha mancato per un pelo. Avevo mangiato Tarallino, il mio amore e non me n'ero accorta!

(ibid, 65-66)

Il suo intervento è sarcastico, derisorio, e in ragione di questo prepotentemente efficace: affronta con l'ironia della saggezza il problema del vegetarianismo da una prospettiva non più ideologica, ma storica. 4 Nel campo di battaglia della cucina si confrontano dunque tre forme di privazione, termometro di tre epoche diverse: gli stenti di Mela (seconda guerra mondiale), la rinuncia di Rosaria (rivoluzioni comuniste) e il disgusto di Carmen (boom economico).

\subsection{La «miopia della rivoluzione»}

Vi è tuttavia un passaggio netto del testo in cui questo livello di conflittualità, in parte fisiologico, cessa di predominare lasciando che si sprofondi verso la feroce e 
dolorosa comunicazione dialettica del disconoscimento del modello, che si reifica - nel dialogo di maggiore tensione della pièce, quando Carmen sta rivelando a Rosaria la sua relazione con Costante - nell'allegoria dello «sciroppo di ciliegie» come tentativo di un dono e rifiuto di esso:

ROSARIA: Ma cosa c'è? Perché te la prendi con me, che t'ho fatto?

CARMEN: Odio il tuo sciroppo di ciliegie, troppo dolce, hai capito, troppo dolce, mi fa vomitare! [...] Mi riempi del tuo sciroppo e non capisci niente.

(MARAINI 2001, 81-82)

Carmen rinfaccia con violenza apparentemente gratuita alla madre il suo continuo, fin dall'infanzia, soprassedere sui pasticci della figlia, giustificandoli, la sua incapacità di rimproverare, di porre un veto, ma soprattutto la rivoluzione, così totalizzante rispetto a prospettive socio-politiche, da aver reso Rosaria cieca nel quotidiano:

Sei cieca. Sei stupida, stupida di una stupidità senza limiti! col tuo idealismo del cazzo, guarda che cosa hai combinato: sei diventata una vittima di te stessa, una vittima mia, una vittima della nonna. [...] Non mi hai mai picchiata. Mai rimproverata. Mai detto niente di niente. Tutto quello che facevo era giusto. [...] e così la bambina è venuta su viziata, pigra, egoista, noiosa, capricciosa. E tutto per colpa tua, per la tua eterna, sbavante, inutile, cretina bontà di madre. (ivi)

Il monologo è caratterizzato da enumerazioni e climax ascendenti, nella funzione di un ritmo rapido e concitato che connota il linguaggio di Carmen come emotivo, di accumulo, indistinto.

Sentendosi offuscata dalle altezze rivoluzionarie materne, la diciannovenne spera forse perversamente di essere vista da Rosaria attraverso Costante, mediante cioè l'ammissione di un pasticcio infantile che si evolve nella colpa di un tradimento. Di certo dichiara la propria inedia, deriva e vacuità etica come conseguenze dell'idealismo, della cecità materna: un peccato poco dopo riaffermato da Mela, quando incontra Rosaria visibilmente scossa, come ragione di una nuova impossibilità di riconoscimento, questa volta nella discendenza:

MELA: E tu non avevi capito niente?

ROSARIA: Niente. 
MELA: Io non so da chi hai preso. Da me, no. [...] Ma, è vero, tu sei quella della rivoluzione, della bontà, della fedeltà. E come puoi capire queste cose così piccine?... (MARAINI 2001, 84)

Rosaria opera una scelta: il suicidio, proprio in cucina, quasi a sancire la morte del focolare domestico; si veste elegante, si trucca, come per un appuntamento galante, accende il gas e si siede. In contrappunto antifrastico, dietro le quinte, si svolge uno sguaiato battibecco tra Mela e Carmen che ipotizzano i nomi da dare al bambino: Bilancia se è femmina, Termometro se è maschio. Due nomi inusuali, ispirati a Mela da un «americano, lì, quello dei petroli» (MARAINI 2001, 86) che aveva chiamato il figlio «Grammofono» (ivi). Questo dialogo, apparentemente secondario, cela un interessante elemento in funzione allegorica: conosciuto realmente un americano "stravagante" che chiamò il figlio Gramophone, l'autrice - in un'intervista del 2003 - spiega di aver utilizzato questo nome assurdo in Buio (1999) per un «bambino infelice della cronaca, che subisce senza capire la violenza degli adulti», (BRENDLER-IODICE 2003, 84-85) prima tra tutte quella dei genitori che gli «appioppano un nome importabile» (ivi). Grammofono è il nome di un «destino infelice» (ivi), dono di una sadica incoscienza, di una madre come Carmen. Terminata questa discussione tra perturbanti risate, Mela irrompe in cucina, nel sogno di morte di Rosaria, e fraintende la situazione; crede infatti che Rosaria si sia vestita così per Costante e le dice, concludendo lo spettacolo:

Ma che fai? Ti prepari a un appuntamento d'amore? Sei bellissima, Rosaria, stai proprio bene... [...] L'hai lasciato tu il forno aperto? Deve essere quella sventata di Carmen...guarda...i rubinetti del gas aperti...roba da ammazzarci tutti...per fortuna c'è lo sciopero...niente gas per oggi e domani... (MARAINI 2001, 86).

Mela esce nuovamente di scena lasciando la figlia inerme. Al di là della beffa finale del destino della donna, emerge significativamente in queste ultime battute l'imbellettamento di Rosaria che ha come conseguenza l'apprezzamento stupito (il primo in tutto il dramma) da parte di Mela e che motiva le sue ragioni non in una solitudine disperata, quanto alla luce di una narrazione che nella pièce si sviluppa parallela, in sordina, dall'apertura: la storia di Chiang Ch’ing, moglie di Mao, arrestata subito dopo la scomparsa del grande leader, ritenuta sovversiva 
nell'ambito di una ridefinizione interna degli equilibri di potere nel partito, condannata a morte nel 1981 dal governo cinese.

La figura di Chiang Ch’ing è il tu privilegiato a cui Rosaria si rivolge lungo l'intero svolgimento del testo in tre monologhis che sviluppano, in dei quadri isolati dalla vicenda principale, una narrazione di secondo grado.

Compagna Chiang Ch'ing, siete accusata di avere usato le guardie rosse come vostro esercito privato; siete accusata di avere fatto una vita da imperatrice, siete accusata di avere portato le gonne corte, di avere messo il rossetto, di avere trasformato il Palazzo del Cielo in un lupanare del capitalismo...Compagna Chiang Ch’ing siete condannata a morte! (MARAINI 2001, 46)

Esordisce così il dramma, come una profezia (si noti la solennità del ritmo paratattico e anaforico). Mentre pulisce per terra Rosaria in questo primo monologo inscena per se stessa la condanna di Chiang Ch’ing, gioca al teatro, immagina e riproduce i dialoghi, vede la realtà che si autorappresenta e la commenta, divenendo nel contempo agente e spettatrice. È come se Rosaria, apparentemente così ancorata alla realtà, all'impegno sociale e al materialismo storico, scivolasse in una doppia dimensione, immaginifica, visionaria alla ricerca del riscatto alle sue frustrazioni.

La figura di Chiang Ch'ing, o meglio l'immagine che Rosaria si crea di Chiang Ch'ing, si delinea di monologo in monologo come una donna fisicamente minuta, bruttina, ipovedente, assolutamente sobria nell'aspetto, di grande rigore e forza etica, incorruttibile e pronta al sacrificio in nome dell'ideologia comunista, di una rivoluzione atta a modificare gli statuti sociali e culturali. ${ }^{6}$ Chiang Ch'ing emerge come un'icona statuaria, avvolta di sacralità, verso cui Rosaria prova un'ammirazione ai limiti della devozione. Del resto l'atmosfera indistinta e rarefatta di queste narrazioni fa percepire l'azione terribilmente vicina e lontana, appartenente a un tempo assoluto, e consegna non eventi, ma valori, contribuendo alla ricezione di Chiang Ch'ing come mito. Tale narrazione si muta nell'ultimo quadro (successivo alla rivelazione di Carmen e all'incontro con Mela, e decisivo nella scelta della propria morte) da mitica in allegorica, mediante l'esperienza del tradimento attraversata nella cecità della passione ideologica: 
ROSARIA: (chiudendo bene le finestre) Compagna Chang Ch’ing, la rivoluzione tradisce, tradisce sempre...Dove sarà la borsa...la...la borsa... (Trova la borsa; si trucca. Si pettina) Mentre il plotone di esecuzione prepara i fucili...noi due...compagna Chiang Ch'ing, che scarpe ti sei messa per affrontare gli aguzzini? Sembrano due barche!...se viene Costante voglio che mi veda tranquilla, non troppo pallida, o troppo ciancicata...Compagna Chiang Ch’ing, fossi in te mi toglierei quelle scarpe di pezza che fanno proprio schifo...mi toglierei quella giacchettina che sembra la giacchetta di un impiegato del catasto...un poco di eleganza, per dio, di fronte alle masse!...una camicia bianca, i capelli ben riavviati, una sigaretta fra le labbra...no, così sembro una diva del cinema, fallita...compagna Chiang Ch'ing, le rivoluzioni tradiscono sempre, come i figli...e noi ce ne fottiamo. La nostra parte l'abbiamo fatta...siamo dei reduci della storia. Dopo di noi arriveranno gli anni della decomposizione politica. Meglio fare un'uscita da regina!

Va ad aprire la chiavetta del gas, si siede impettita su una sedia e aspetta la morte. (MARAINI 2001, 85)

Il monologo mantiene una partitura emblematica caratterizzata da forti cesure collocate nei continui rimandi analogici tra le vicende delle due donne - rimandi non esplicitati, ma organizzati secondo lo stream of consciousness e riconoscibili solo mediante le variazioni ritmiche dettate dalle cesure - e dal ritorno di una sintassi paratattica, ma pacata, scandita solennemente da apostrofi ed esclamative. Emblematica anche la rapida e frequente alternanza binaria dei pronomi personali (espliciti o sottintesi) io e tu, sostituiti alla fine dal collettivo noi.

Come Chiang Ch'ing viene tradita dai compagni rivoluzionari, così Rosaria dalla figlia; entrambe sono cieche per idealismo, due «reduci della storia» (ivi), sia essa politica o personale. Tra le due sfere, infatti, non vi è distinzione, in quanto emanazioni della medesima struttura etica, di un modello ormai in «decomposizione» (ivi). Il gesto di Carmen non ha una valenza solo privata: il tradimento della madre (generatrice e creatrice) è un atto politico: frutto e causa della frammentazione morale, quindi sociale, quindi culturale, di cui la politica dovrebbe essere custode. Così ogni rivoluzione è figlia, creatura e dedizione di chi da viscerali profondità umane la concepisce, genera, partorisce e sostenta. A tradire Chiang Ch’ing e Rosaria è la Storia, che nei comunisti e in Carmen si fa evento, si fa scissione. Come Chiang Ch'ing, Rosaria decide di affrontare la morte e di sottrarsi al tempo della degenerazione. Le due vicende si sovrappongono e Rosaria, in questa sua ultima visione, incontra e si rivolge direttamente a Chiang 
Ch’ing, smette di impersonarla e la riconosce come sua immagine speculare, come suo doppio. Esorta, dunque, con veemenza la compagna a dismettere i costumi della privazione e del rigore per vestirsi da regine, ad abbandonare, quindi, gli abiti dimessi della rivoluzione, pronte a indossare la maschera che renderà epica la loro morte. Trucco e abiti infatti, nel linguaggio teatrale, assumono una funzione semantica, il valore della maschera: questa ha accesso al perenne ciclo di nascita e morte, ha il potere di rendere fisso un volto, un carattere e il suo valore che diviene, come l'allegoria, universale e si consegna a chiunque lo indossi. Inoltre la maschera come immagine di morte veicola la volontà di Rosaria, e del suo più riuscito e realizzato alter ego, di divenire un fantasma della storia - si consideri l'influenza del teatro del Nō, così impresso nella memoria di Maraini, dove il viso mascherato rappresentava il defunto. $7 \mathrm{Ma}$ Chiang Ch’ing non muore, non nel 1981, e non muore nemmeno Rosaria, rendendo pirandellianamente umoristica la sua veste finale, quasi una parodia del suo nobile alter ego.

Ciò che rimane sono i figli di Carmen, gli anni della «decomposizione politica» (ivi) (i nostri) e le maschere.

\subsection{Il teatro: la rivoluzione di una memoria allucinata}

Vive un discorso fluido e sotteso (ma determinato) all'intera pièce, ed è una riflessione metateatrale. Questa scorre nel testo mediante diversi canali, di cui due sono certo i più copiosi: il parlare per citazioni di Mela e le visioni di Rosaria. Più volte, infatti, Mela risponde e si inserisce nei dialoghi quotidiani con figlia e nipote utilizzando stralci della Locandiera di Goldoni, dissemina, inoltre, il testo con "siparietti" in cui ripete e interpreta per gioco passi sempre goldoniani e della Nemica. L'intera commedia, del resto, vive attraverso gli aneddoti della vita in tournée, ricordi vivi sia nella memoria di Mela che in quella di Rosaria che da bambina girava l'Italia con la madre (anche in questo caso la figura maschile è assente): 
ROSARIA: Io, da sotto in su, a guardare quelle belle camicie svolazzanti e mi incantavo...ma perché non ho fatto il teatro non lo so.

MELA: Perché ti sei innamorata della politica. E ti pareva che il teatro non fosse abbastanza politico. E ti sei messa a correre per il mondo inseguendo la rivoluzione... (MARAINI 2001, 78)

In questo eloquente scambio di battute Mela esprime il velato risentimento per il tradimento della figlia di una memoria matrilineare che dalla maschera conduce a Mela, di una storia fatta di lotta e rivoluzioni sebbene non di propaganda basti pensare alla riforma goldoniana e ai sacrifici sovversivi che comunque Mela, evidentemente ragazza madre, deve aver compiuto per essere, negli anni '40/'50, una suggeritrice di teatro in giro sola con una bambina. Ma se il tradimento di Carmen nasce da un infantile nichilismo al confine con l'egotismo, quello di Rosaria è ideologico. Echeggia, infatti, una domanda che si pone Maraini stessa alle soglie della sua attività teatrale, nella Premessa al programma di sala della prima di Recitare (11 marzo 1969, Roma, circolo La Fede):

C'è qualche possibilità per il teatro di intervenire nel cambiamento di una società? $\mathrm{O}$ il teatro non può essere che il riflesso passivo di una data situazione sociale? (WEINBERG 1998, 24).

Il ripresentarsi della riflessione nel 1981 mantiene vivo l'intendimento della ricerca teatrale e sociale degli inizi, che in Mela si arricchisce di implicazioni storiche e culturali. Rosaria infatti ricorda con Carmen di aver assistito a Parigi al balletto La ragazza dai capelli bianchi, un'opera della Rivoluzione Culturale Cinese attraverso cui Mao e Chiang Ch’ing demolivano i vecchi statuti artistici, culturali e morali. Rosaria riconosce al teatro di poter essere rivoluzionario, ma ma non a quello italiano, e reprime la sua passione per il teatro per ribellarsi a un canone vecchio e chiuso in se stesso, che è frammento metonimico di un più vasto codice morale. Ne avalla uno nuovo e inclusivo, quello politicamente impegnato, quello sperimentale per cui Maraini ha tanto combattuto lungo un percorso in cui gli anni Ottanta, quelli di Mela, segnano la fine dell' «ottimismo» del «periodo militante» e la consapevolezza di una «mancata utopia» (WEINBERG 2003, 47).

Del resto, lo si diceva, all’interno dell'azione principale (la vicenda di Mela, Rosaria e Carmen) si animano i sogni e i ricordi di Rosaria, che aprono nella 
scena un varco dove dispiegare memoria, fantasticazione e denuncia sociale. A queste zone di testo Maraini consegna parte della sua riflessione metateatrale, in quella performance di perfetta verosimiglianza delle allucinazioni di Rosaria ottenuta recuperando, mediante

parole raccolte ascoltando o emanate dai fantasmi che visitano la 'stanza' della mente, una sensibilità di tipo aristotelico, per cui il segno verbale non deve essere tanto 'vero' - brulicante e sporco frammento del parlato o di intuizioni irriflesse - ma 'verosimile', veicolo tra l'identità dell'emittente retorica e il mondo di nessi che restaura in forma testuale la complessa realtà delle cose» (GUCCINI 2000, 83-84).

\section{Mela, la Madre e il Canone}

Vi è dunque Mela, una commedia in due atti dove una parola nuova, quotidiana, 8 in grado di «spostare il conflitto dal piano dell'azione a quello della dialettica verbale» (TAFFON 2010, 210), dà voce alle donne e lascia circolare in moto perpetuo la dialettica tra agire presente e canone, nella complessità di accezioni che quest'ultimo ammette a partire dal suo preciso senso etimologico dal greco $\kappa \alpha v \omega ́ v$ (asta, bastone dritto e lungo). Ci si allinea qui, per una precisazione funzionale a queste note, alla duplicità di prospettiva individuata da Luperini nell'uso del termine:

dal punto di vista delle opere (potremmo dire: a parte obiecti) e della loro influenza. È l'insieme di norme (retoriche, di gusto, di poetica ecc.), tratte da un'opera o da un gruppo di opere omogenee, che fonda una tradizione e che perciò determina l'elaborazione di una serie di altre opere. Nella seconda accezione il canone è considerato dal punto di vista dei lettori e del pubblico, dunque della ricezione (potremmo parlare, in questo caso, di un canone visto a parte subiecti): indica la tavola dei valori prevalente (LUPERINI 2000, 11).

A parte subiecti, Dacia Maraini ammette con lucidità:

C'è una difficoltà dal punto di vista dell'istituzione letteraria ad accettare come prestigiosa la letteratura femminile. È sempre considerata alla stregua del mercato [...], ma per essere apprezzati dalla critica e da coloro che stabiliscono i valori per le prossime generazioni dal punto di vista del modello, cioè dell'esemplarità, i libri delle donne vengono castigati (WEINBERG 2003, 51). 
È proprio questa consapevolezza (quella discriminatoria) a determinare la posizione dell'autrice rispetto al canone a parte obiecti. All'interno del panorama letterario italiano, è nell'atto stesso della sua scrittura che Maraini interagisce in modo dialettico col canone, perché drammaturga e regista in uno stesso tempo, perché intenzionata a «ripristinare integralmente la soggettività della donna e [...] ricostruire uno spazio e un tempo che le sono propri» (WEINBERG 2003, 46) coinvolgendo «la storia vista nel suo insieme di fenomeni [...] che hanno condizionato la realtà della donna fino ai giorni nostri» (ivi). Un'operazione che porta avanti, del resto, in un secolo in cui il canone letterario subisce una trasformazione che contrappone il valore estetico a quello sociale, a seguito della frattura di «quella solidarietà etica prima che estetica, che univa produttori e consumatori», che comporta la rottura del «nesso di etica ed estetica» (Guglielmi 2000, 36).

Ma si potrebbe aggiungere una terza sfaccettatura del problema canone in Maraini, che potremmo definire a parte auctoris, o meglio, relativa al concetto di autorità a esso legato: infatti «non c'è canone, senza un'autorità che lo ponga» (ONOFRI 2001, 8), sia esso letterario, politico, culturale o etico. E in Mela vibra come sostegno del testo una riflessione sull'ortodossia, sia essa letteraria, politica, culturale o etica; e cos'altro è questa, se non il canone: uno schema, un modello che «isola valori, esempi, modelli dotati di un'autorità metastorica» (BERARDinElli 2000, 94), che si offre come definito e concluso e che invece si implementa di elementi successivi. Questi non possono essere del tutto eterogenei e fanno riferimento a un nucleo concettuale condiviso che lentamente si sedimenta come "opinione corretta" ( $o \theta \theta o \varsigma-\delta o ́ \xi \alpha$ ) epurando progressivamente ciò che non vi si conforma.

Politicamente è la vicenda di Chiang Ch’ing a rappresentare la ciclicità storica dei moti rivoluzionari, in quella sua condanna per essere stata infedele alla rivoluzione, per «aver messo il rossetto» e «portato le gonne corte» (MARAINI 2001, 46). E ancora Mela, col suo evidente anticonformismo, libera dai dogmi culturali di una società che vorrebbe, come Carmen, le nonne chiuse in casa a «occuparsi dei nipoti» (MARAINI 2001, 61). E infine Carmen e il suo tradimento 
della madre. Quattro dinamiche che concludono il loro circuito dialettico nella stasi finale di Rosaria, sola e con indosso una maschera, su cui si chiude il sipario.

Questa allude alla smorfia dei morti, agli spiriti della casa e quindi al passato personale come ipostasi che si tramuta in una visione reificata dei vivi. Saper indossare la maschera: il canone, quale norma dell'autorialità, implica un processo identitario che però il più delle volte è frutto del rapporto dinamico e dialettico fra i diversi autori e i diversi modelli culturali. Ciò significa che il canone necessita del tradimento per permettere alla ciclicità generativa di tornare su se stessa, esso è tradito e traditore. Così Rosaria, subìto il tradimento di Carmen, è pronta a vestire i panni di Mela, la sua eleganza, la sua maschera, accogliendo in sé, nel suo essere presente, anche il passato. Può affrontare la morte solo una volta pagato il contrappasso per la sua precedente colpa, in fondo la medesima. Mela, Rosaria e Carmen rinnegandosi si affermano come singole individualità e identità, e alla fine come discendenza declinando un processo che si mostra pur sempre

un male necessario e intrascendibile, e soprattutto [...] un principio generativo, idoneo a conferire una nuova forma e a suscitare processi di trasformazione (CURI 1999, 12-13).

Le tre donne sperimentano l'«esperienza della separatezza» dal Canone (ZACCARO 2008-2009, 211), la caduta dall'albero: mela. Se il teatro crea, come atto predeterminato e premonizione, una relazione con lo «sguardo di Dio» (MARAINI 2000, VI), il posto che «ha la madre di tutte le madri in questa cosmogonia» (ivi) è quello della mela: simbolo del tradimento del primo precetto del canone del Grande Autore - un «corpus» indiscutibilmente «chiuso» (BRIOSCHI 2000, 157) - , del primo atto di trasgressione, e anche il simbolo del frutto che, reciso, vive, matura e semina.

Qui il canone di Maraini è donna. È la storia dimessa di generazioni che ubbidiscono al silenzio e a cui l'autrice si ribella senza violenza, priva di «risentimento» (FERRONI 1998, 79), cercando di destrutturarlo tramite un dono, la voce, la parola: la trasformazione dell'esperienza del tradire in quella del tradurre. Mediante la memoria, nel momento stesso in cui rinnega il canone, 


\section{Maraini lo accoglie dandosi la possibilità del mutamento, di risolvere la dialettica, affidando e restituendo alla letteratura una nuova «fede» (ibid., 82).}

Come si vede le memorie sono tante e tutte fruttuose. Il mio sentimento di inadeguatezza si è temperato col tempo, anche attraverso il recupero delle memorie sommerse (MARAINI 2010, 389).

\section{Note}

${ }^{1}$ Si segnala che il presente contributo è stato oggetto della comunicazione tenuta da E. Ghirlanda al Congresso Internazionale "Ausencias. La reconstrucción del canon literario en Europa y las escritoras" (Siviglia, 11-13 dicembre 2017). Il contributo è consultabile in Escritoras italianas fuera del canon, a c. di Daniele Cerrato, Asociación Cultural Benilde Mujeres\&Culturas, Culturas\&Mujeres Sevilla 2017, pp. 198-228.

2 Il testo debutta nel gennaio del 1982 ad Ancora, presso il teatro comunale Rossini, come produzione del Collettivo Isabella Morra (regia di Antonio Calenda; Elsa Merlini nel ruolo di Mela, Saviana Scalfi in quello di Rosaria e Chiara Salerno in quello di Carmen; scena di Uberto Betacca; costumi di Ambra Danon e musiche di Mario Pagano).

3 Medesimo procedimento si rintraccia in Stravaganza dove «la scrittrice ribalta il topos del focolare domestico come accoglienza, dove si nutre e si mantiene viva la famiglia, facendolo diventare negazione della stessa» (TAURO 2008, 195).

$4 \mathrm{Si}$ ricorda a tal proposito la rilevanza data da Maraini proprio in quel suo saggio sull'Inadeguatezza all'esperienza della fame nel campo di concentramento: «Quando la fame è così prepotente, non si riesce a pensare ad altro. Tutto il giorno parlavamo di cibo. Il cibo era diventato un'ossessione, un incubo, era diventato in nostro mito quotidiano» (MARAINI 2010, 390).

5 A queste narrazioni visionarie se ne aggiungono due narrate come ricordi della guerra in Bolivia, in cui Rosaria combatte al fianco di Chopa, un guerrigliero alla fine ucciso a colpi di mitragliatrice.

6 «E lei, dietro quelle due lenti grosse come due fondi di bottiglia, con quella piccola testa di tartaruga...», «Lei, piccola, nana, brutta, vestita di blu, con i capelli grigi a paggetto e la faccia rugosa...Che fegato!» (MARAINI 2001, 46); «La compagna Chiang Ch'ing si alza, con la sua faccetta raggrinzita, gli occhiali come due fondi di bottiglia...La miopia della rivoluzione...»; "Voglio vedere in faccia i miei assassini, dice» (ibid., 52); "La compagna Chiang Ch'ing con la testa circondata di fiammelle d'oro...e il compagno Mao con le sue scarpe di pezza...che calpestavano le vecchie regole, i vecchi sentimenti, le vecchie morali...paf...paf...» (ibid., 53).

7 «Da bambina sono stata portata a una rappresentazione del teatro Nō. [...] Poi la lentezza dei gesti, l'eleganza delle maschere. C'era qualcosa di arcano e di misterioso in tutta la rappresentazione che mi attirava nel momento stesso in cui mi sfuggiva. Anche il fatto che fossero sempre dei morti che parlavano coi vivi era una cosa che mi inquietava. Ma, ecco, questa è una cosa che ho imparato e mi è servita anche dopo: i morti non sono figure temibili da cui sfuggire, ma persone poetiche a cui chiedere pareri e compagnia. Al teatro Nō mi sono ispirata quando ho scritto Il Manifesto, storia di una ragazza raccontata dalle sue amiche morte» (MARAINI-MURRALI 2013, 20-21).

8 Caratterizzata da fenomeni linguistici, come ad esempio la dislocazione a destra dei complementi, frasi scisse con struttura pseudo-relativa; la scelta, inoltre, di un italiano standard colloquiale o lo stilema, tipico di Maraini, di chiuse con similitudini che comparano uomini e animali (TAFFON 2010, 212-215). 


\section{Bibliografia}

\section{BARTHES 1998}

Roland BARTHES, Scritti, Società, Testo, Comunicazione, Torino, Einaudi, 1998.

\section{BERARDINELLI 2000}

Alfonso BERARDINELLI, Alla ricerca di un canone novecentesco, in Il canone letterario del Novecento italiano, Nicola Merola (a c. di), Soveria Mennelli, Rubbettino, 2000, pp. 93-103.

\section{BRENDLER-IODICE 2003}

Andrea BRENDLER-Francesco IODICE, Intervista a Dacia Maraini sui nomi, in «Italian Quarterly», XL, 155-156 (2203), pp. 81-89.

\section{BRIOSCHI 2000}

Franco BRIOSCHI, Paradossi del canone, in Il canone letterario del Novecento italiano, Nicola Merola (a c. di), Soveria Mennelli, Rubbettino, 2000, pp. 153-168.

\section{CRUCIATA 2003}

Maria Antonietta CRUCIATA, Dacia Maraini, Fiesole, Cadmo, 2003.

\section{CURI 1999}

Umberto CURI, Pensare la guerra. L'Europa e il destino della politica, Bari, Edizioni Dedalo, 1999.

\section{GUCCINI 2000}

Gerardo GuccINI, Voci insistenti, recitanti, angeliche. Il teatro "sentito" di Dacia Maraini, in Dedica a Dacia Maraini, Claudio Cattaruzza (a c. di), Trieste, Lint, 2000, pp. 73-89.

\section{GUGLIELMI 2000}

Guido Guglielmi, Canone classico e canone moderno, in Il canone letterario del Novecento italiano, Nicola Merola (a c. di), Soveria Mennelli, Rubbettino, 2000, pp. 33-54.

FERRONI 1998

Giulio FERroni, Al di là dal canone, in «Allegoria», X, 29-30 (1998), pp. 75-82.

\section{LUPERINI 2000}

Romano LUPERINI, Il canone del Novecento e le istituzioni educative, in Il canone letterario del Novecento italiano, Nicola Merola (a c. di), Soveria Mennelli, Rubbettino, 2000, pp. 11-21.

MARAINI 2000

Dacia MARAINI, Fare teatro, Milano, Rizzoli, 2000.

MARAINI-MURRALI 2013

Dacia MARAINI-Eugenio MURRALI, Il sogno del teatro, Milano, Bur, 2013. 
MARAINI 2001

Dacia MARAINI, Maria Stuarda e altre commedie, Milano, Bur, 2001.

MARAINI 2010

Dacia MARAINI, Inadeguatezza, in Scrittura civile. Studi sull'opera di Dacia Maraini, Juan Carlos de Miguel y Canuto (a c. di), Roma, Giulio Perrone Editore, 2010, pp. 377-389.

MARIANI 2000

Laura MARIANI, Dacia Maraini e il "selvatico mistero" della scena, in Dedica a Dacia Maraini, Claudio Cattaruzza (a c. di), Trieste, Lint, 2000.

MARINELLI-MATASSA 2008

Gioconda MARINELLI-Angela MATASSA, Dacia Maraini in scena con Marianna, Veronica, Camille e le altre, Pescara, Ianieri Editore, 2008.

ONOFRI 2001

Marco ONOFRI, Il canone letterario, Roma-Bari, Laterza, 2001.

TAFFON 2010

Giorgio TAFFON, Parola e dialogo (monologo) nel teatro di Dacia Maraini, in Scrittura civile. Studi sull'opera di Dacia Maraini, Juan Carlos de Miguel y Canuto (a c. di), Roma, Giulio Perrone Editore, 2010, pp. 201-221.

TAURO 2008

Francesco TAURo, Dalla tavola al testo, in «La Nuova ricerca», 17-18 (2008), pp. 189196.

WEINBERG 1998

Grazia Sumeli WeInberg, Dacia Maraini e il teatro femminista come modello di trasgressione, in «Studi d'italianistica nell'Africa Australe», XI, 3 (1998), pp. 20-31.

WEINBERG 2003

Grazia Sumeli WEINBERg, Il femminismo con un altro nome: intervista a Dacia Maraini, in «Studi d'italianistica nell'Africa Australe», XVI, 2 (2003), pp. 44-58.

ZACCARO 2008-2009

Vanna ZACCARO, Le scritture di donne ai margine del canone?, in "La Nuova ricerca», XVII-VIII, 17-18 (2008-2009), pp. 209-214. 